\title{
MicroRNA-424 may function as a tumor suppressor in endometrial carcinoma cells by targeting E2F7
}

\author{
QUAN LI, XIANG-MEI QIU, QING-HAN LI, XIAO-YAN WANG, LI LI, \\ MIN XU, MIN DONG and YAN-BING XIAO \\ Department of Gynaecology, The First Affiliated Hospital of Zunyi Medical College, \\ Zunyi, Guizou 563000, P.R. China
}

Received August 22, 2014; Accepted December 8, 2014

DOI: $10.3892 /$ or.2015.3812

\begin{abstract}
MicroRNAs (miRNAs) are frequently dysregulated in human cancers and can act as potent oncogenes or tumor suppressor genes. Aberrant expression of miR-424 has been identified in some types of cancer, however, its expression and potential biologic role in endometrial cancer are remains to be determined. In the present study, we demonstrated that miR-424 was downregulated in human endometrial cancer and suppressed growth of the human Ishikawa and HEC-1B endometrial cancer cell lines. Bioinformatics analysis indicated that E2F7 was a putative target of miR-424. In a luciferase reporter system, we confirmed that E2F7 was a direct target gene of miR-424. Furthermore, knockdown of E2F7 inhibited Ishikawa and HEC-1B cell growth. These findings indicate that miR-424 targets the E2F7 transcript and suppresses endometrial cancer cell growth, suggesting that miR-424 has a tumor suppressive role in human endometrial cancer pathogenesis.
\end{abstract}

\section{Introduction}

MicroRNAs (miRNAs) are a class of small non-coding RNAs consisting of 19-24 nucleotides that are important in the negative regulation of gene expression. miRNAs bind to partially complementary sequences in the 3'-untranslated region (UTR) of specific target mRNA, resulting in mRNA degradation or translation inhibition $(1,2)$. Abnormal expression and the loss of the dynamic balance between oncogenes and tumor suppressor genes typically lead to tumor formation and the development of cancer (3). As an important regulator of gene expression, the abnormality of miRNAs has been observed in various types of cancer and may be involved in modulating cancer cell behaviors $(4,5)$. Considerable evidence has shown

Correspondence to: Dr Yan-Bing Xiao, Department of Gynaecology, The First Affiliated Hospital of Zunyi Medical College, 149 Dalian Road, Zunyi, Guizou 563000, P.R. China

E-mail: xiaoyanbing822@163.com

Key words: endometrial cancer, microRNA, miR-424, E2F7 that miRNAs are important regulators in diverse biological processes of cancer, such as cell proliferation (6), apoptosis (7), angiogenesis (8), cell differentiation (9), adhesion and metastasis (10). These data emphasize the importance of miRNAs in cancer development and provide new insights into understanding the molecular mechanism of tumorigenesis.

Endometrial cancer is the second most prevalent cancer among women, following breast cancer. In the USA, it is estimated that 624,890 women have been previously diagnosed with cancer of the uterine corpus, and an additional 52,630 cases are expected to be newly diagnosed in 2014 (11). The development of endometrial cancer is a multistep process with accumulation of genetic and epigenetic alterations (12). Microarray studies have identified a number of miRNAs, such as miR-27 (13), miR-203 (14), miR-205 (15) miR-141, and miR-200 (16), which were upregulated, whereas, miR-30c (17), miR-101 (18), miR-449 (19), miR-143 and miR-145 (16) were downregulated in endometrial cancer. However, the precise role of miR-424 in the carcinogenesis of endometrial cancer has yet to be determined.

In the present study, we investigated the expression of miR-424 in human endometrial cancer and paired adjacent normal pancreatic tissues, and explored the effects of miR-424 on cell growth and cycle progression in vitro. Furthermore, E2F7 was identified as a direct target of miR-424 and confirmed by the luciferase reporter system. Our results suggested that miR-424 directly targets E2F7 and negatively regulates cell proliferation and cycle progression in Ishikawa and HEC-1B cells, suggesting that miR-424 has as a tumor suppressive role in human endometrial cancer pathogenesis.

\section{Materials and methods}

Patient tissue specimen. Eleven pairs of tissues from 11 endometrial cancer patients, consisting of human endometrial cancer tissue and matched adjacent normal tissue obtained from the same patient, were used in the present study. The matched normal tissue was extracted from the distal end of the operative excisions, far from the tumor. The samples were received from the First Affiliated Hospital of Zunyi Medical College (Guizou, China).

Cell culture and transfection. The human endometrial cancer cell line Ishikawa and HEC-1B were purchased from 
ATCC (Manassas, VA, USA). The cells were maintained in Dulbecco's modified Eagle's medium supplemented with $10 \%$ fetal bovine serum (both from Gibco, Carlsbad, CA, USA) in a humidified incubator at $37^{\circ} \mathrm{C}$ with $5 \% \mathrm{CO}_{2}$. The transfection was performed using the Lipofectamine 2000 reagent (Invitrogen-Life Technologies, Carlsbad, CA, USA) according to the manufacturer's instructions.

RNA extraction and RT-PCR. RNA was extracted from patient tissue specimen or HEC-1B cells $48 \mathrm{~h}$ after transfection using TRIzol reagent (Invitrogen, Shanghai) according to the manufacturer's instructions. Small RNA (5 $\mu \mathrm{g})$ was reverse transcribed into cDNA using M-MLV reverse transcriptase (Promega, Madison, WI, USA) with the specific primers. The cDNA was used as a template to amplify mature miR-424 or the U6 snRNA endogenous control by PCR. The PCR was performed as follows: $94^{\circ} \mathrm{C}$ for $3 \mathrm{~min}$, followed by 40 cycles of $94^{\circ} \mathrm{C}$ for $30 \mathrm{sec}, 50^{\circ} \mathrm{C}$ for $30 \mathrm{sec}$ and $72^{\circ} \mathrm{C}$ for $30 \mathrm{sec}$. The RT-PCR was performed using SYBR Premix Ex Taq (Takara, Japan) on the iQ5 real-time PCR detection system (Bio-Rad, Hercules, CA, USA). The relative expression of miR-424 was defined as follows: quantity of miR-424/quantity of U6 within the same sample. Briefly, a cDNA library was generated through reverse transcription using M-MLV reverse transcriptase (Promega) with large RNA $(5 \mu \mathrm{g})$. The cDNA was used to amplify the $E 2 F 2, E 2 F 3, E 2 F 4, E 2 F 4, E 2 F 5, E 2 F 6, E 2 F 7$ and the $\beta$-actin genes, which served as an endogenous control. The PCR was performed as follows: $94^{\circ} \mathrm{C}$ for $3 \mathrm{~min}$, followed by 40 cycles of $94^{\circ} \mathrm{C}$ for $30 \mathrm{sec}, 58^{\circ} \mathrm{C}$ for $30 \mathrm{sec}$ and $72^{\circ} \mathrm{C}$ for $30 \mathrm{sec}$. RT-PCR was performed as described above, and the relative gene expression level was defined as: quantity of gene/quantity of $\beta$-actin within the same sample.

Cell viability and proliferative capacity assay. To determine cell viability and proliferative capacity, cells were examined using the 3-(4,5-dimethylthiazol-2-yl)-2,5-diphenyltetrazolium bromide (MTT) and colony formation assays as previously described $(20,21)$. Ishikawa and HEC-1B cells, following transfection, were seeded in 96-well plates at a density of $8 \times 10^{3}$ cells/well. At different time points after transient transfection, the cells were incubated with $10 \mu \mathrm{l}$ MTT (at a final concentration of $0.5 \mathrm{mg} / \mathrm{ml}$ ) at $37^{\circ} \mathrm{C}$ for another $4 \mathrm{~h}$. The medium was then removed, and the precipitated formosan was dissolved in $100 \mu \mathrm{l}$ dimethyl sulfoxide (DMSO). After agitation for $20 \mathrm{~min}$, the absorbance at $570 \mathrm{~nm}$ (A570) was detected using a uQuant Universal Microplate Spectrophotometer (BioTek Instruments, Inc., Winooski, VT, USA).

For the colony formation assay, the number of viable cell colonies was determined after 15 days following inoculation of 150 cells/well in triplicate in 12 -well plates. The cells were stained with crystal violet. The rate of colony formation was calculated using the equation: colony formation rate $=$ (number of colonies/number of seeded cells) x $100 \%$.

Cell apoptosis and cell cycle assay. The apoptotic ratios of cells were determined with the Annexin V-7-ADD apoptosis detection kit (Roche, Switzerland). Briefly, $48 \mathrm{~h}$ after transfection, the cells were collected and washed twice with cold PBS buffer, resuspended in $200 \mu \mathrm{l}$ of binding buffer, incubated with $20 \mu \mathrm{l}$ of Annexin V-R-PE for $20 \mathrm{~min}$ in an ice bath in the dark, and 7-AAD $10 \mu \mathrm{l}$ prior to analysis by flow cytometry. The cells treated with DMSO were used as the negative control.

Following transfection for $48 \mathrm{~h}$, the cells were collected and fixed with $70 \%$ ethanol, stained with propidium iodide (PI), and analyzed by flow cytometry. The data were analyzed with CellQuest Pro software (BD Biosciences, San Jose, CA, USA). The experiments were repeated at least three times.

Western blot analysis. Total cell extracts were extracted using RIPA buffer. Proteins were separated by $10 \%$ SDS-PAGE, and proteins of interest were detected using the appropriate antibodies. Rabbit anti-human GAPDH antibody rabbit anti-human E2F7 antibody were obtained from Abcam (Cambridge, UK).

Targetprediction and luciferase reporter assays. Based on bioinformatic prediction (TargetScan, RNA22 and microrna-org), E2F7 was selected as the candidate target of miR-424. The 3'UTR segments of E2F7 containing putative binding sites for miR-424 were obtained by PCR and inserted into the pmirGLO vector. The wild-type reporter construct pmirGLO/E2F7-3'UTR and the mutant reporter construct pmirGLO/E2F7-3'UTR mut, in which the site of perfect complementarity to miR-424 was mutated (UGCUGC $\rightarrow$ UCGGCC) using site-directed mutagenesis PCR, were used for miRNA functional analysis. Wild-type and mutant insertions were confirmed by DNA sequencing. For the luciferase reporter experiments, HEC-1B cells were co-transfected with the miR-424 mimics or as-miR-424 in a 48-well plate followed by the pmirGLO/E2F7-3'UTR reporter vector or the pmirGLO/E2F7-3'UTR mut. Firefly luciferase and Renilla luciferase levels were measured at $48 \mathrm{~h}$ after transfection. Each experiment was repeated at least three times.

Statistical analysis. Data were presented as the means \pm standard deviation (SD). $\mathrm{P} \leq 0.05$ was considered to indicate a statistically significant result using the Students-NewmanKeuls tests.

\section{Results}

Depressed expression of miR-424 in endometrial cancer. To determine the role of miR-424 in endometrial cancer development, we examined the expression of miR-424 in five endometrial cancer samples and three adjacent normal tissues by semi-quantitative RT-PCR (Fig. 1A). Furthermore, miR-424 expression was significantly downregulated in endometrial cancer tissues as compared to matched normal tissues by RT-PCR (Fig. 1B).

miR-424 suppresses the long-term proliferation of the Ishikawa and HEC-1B endometrial cancer cell lines. To determine the role of miR-424 in tumor cell proliferation, the miR-424 mimics were used to overexpress miR-424 expression in Ishikawa and HEC-1B cells (Fig. 2A). Using an MTT assay, the overexpression of miR-424 was demonstrated to suppress viability in Ishikawa and HEC-1B cells (Fig. 2B and C). The colony formation rate of Ishikawa and HEC-1B cells transfected with miR-424 mimics, was significantly reduced as compared to that of the control group (Fig. 2D). These results indicated that miR-424 suppressed the ability of Ishikawa and HEC-1B cells to proliferate. 
A

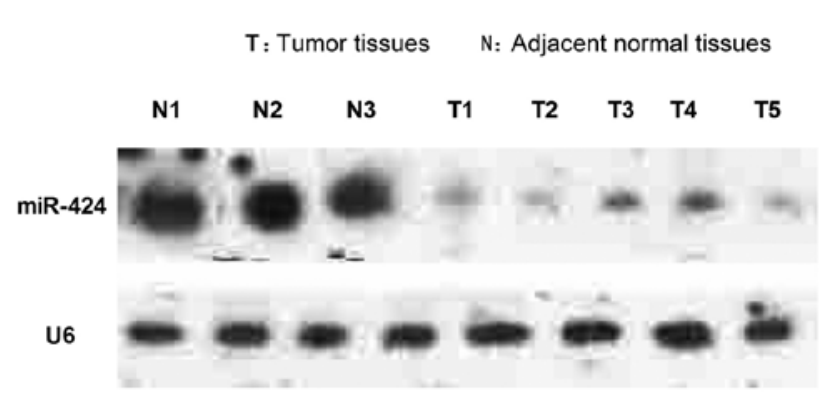

B

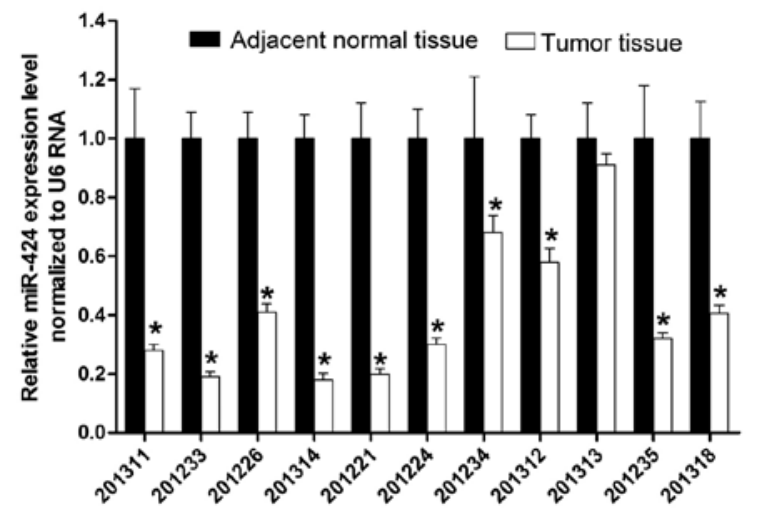

Figure 1. Depressed expression of miR-424 in endometrial cancer. (A) Semi-quantitative RT-PCR analysis of miR-424 expression. (B) RT-PCR analysis of miR424 expression. Fresh tissue samples from endometrial cancer patients and from patients with benign breast diseases were obtained from the surgery room. Total RNA was extracted from the tissues and subjected to semi-quantitative RT-PCR and real-time PCR analysis, showing significant downregulation ( $\mathrm{P}<0.05$ ).

A

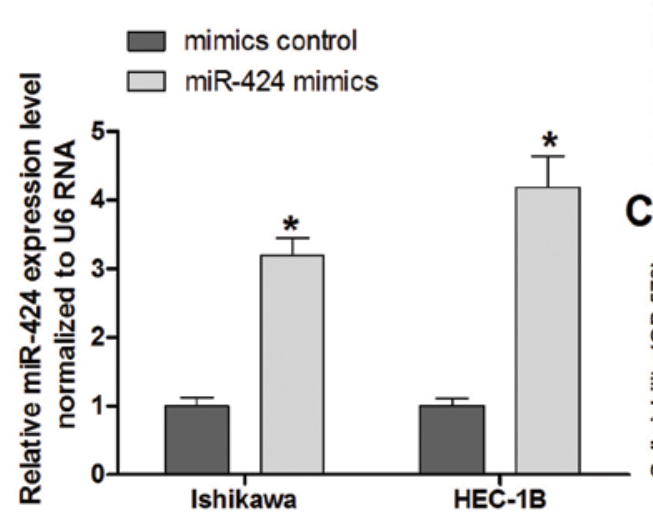

B

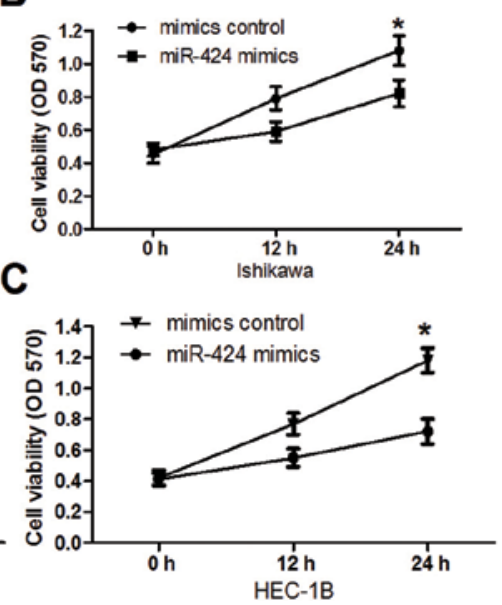

D

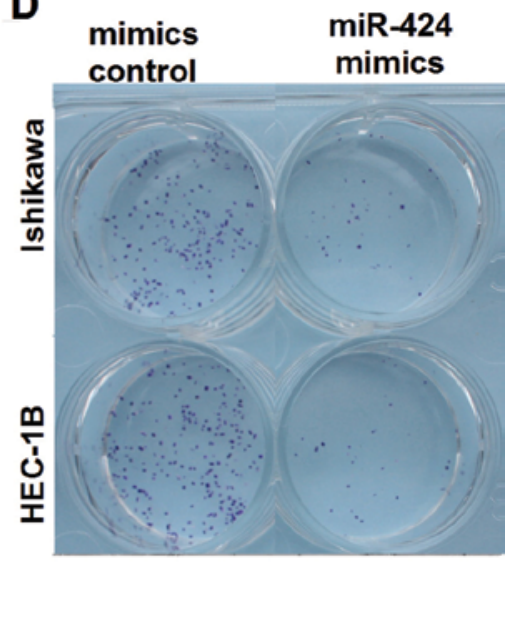

Figure 2. miR-424 suppresses the long-term proliferation of the endometrial cancer cell line. (A) Measurement of miR-424 expression levels by RT-PCR. Small RNA was extracted from Ishikawa and HEC-1B cells transfected with miR-424 mimics or mimics control, and U6 snRNA served as an endogenous control. The relative miR-424 expression level (means \pm SD) is shown ( $\left.{ }^{*} \mathrm{P}<0.05\right)$. (B and C) Cell viability was detected through MTT assay. After Ishikawa and HEC-1B cells were transfected with the miR-424 mimics or mimics control, the MTT assay was used to determine the relative cell growth activity at 0,12 and $24 \mathrm{~h}$ post-transfection. ( $\mathrm{P}<0.05)$. The relative cell growth activity was normalized to the growth activity of Ishikawa and HEC-1B cells in the control groups. (D) Effect of miR-424 on cell proliferation as evaluated by a colony formation assay. Ishikawa and HEC-1B cells transfected with miR-424 mimics or mimics control were seeded in 12-well plates. On the 8th day after seeding, the number of colonies was counted.

miR-424 initiates endometrial cancer cell line $S$ phase arrest and promotes apoptosis. Flow cytometry showed that the percentage of S-phase Ishikawa and HEC-1B cells markedly increased in the miR-424 mimics-transfected group, which was higher than that in NC, suggesting that miR-424 initiated S-phase arrest (Fig. 3A and B). The fluorescence activated cell sorting (FACS) analysis showed that the forced expression of miR-424 led to endometrial cancer cell apoptosis. The percentage of total apoptotic cells (early apoptotic + late apoptotic) significantly increased by $18.9 \%$ in response to miR-424 overexpression compared to cont-miR overexpression in Ishikawa cells (Fig. 3C). A $22.9 \%$ increase in the number of apoptotic cells was observed in the HEC-1B cells with miR-424 overexpression compared to cont-miR (Fig. 3D). These data demonstrated that miR-424 may inhibit proliferation by inducing $\mathrm{S}$-phase arrest and promote apoptosis in endometrial cancer.
miR-424 directly targets transcription factor E2F7. Based on the miR-424-induced suppression of proliferation in the endometrial cancer cell lines, we hypothesized that miR-424 inhibited the malignancy of endometrial cancer cells by regulating oncogenes and/or genes involved in cell proliferation or apoptosis. Thus, bioinformatic analyses (TargetScan, RNA22 and microrna.org) were used to identify potential target genes of miR-424 (Fig. 4A). Quantitative analysis indicated that E2F7 expression was significantly decreased in HEC-1B cells transfected with miR-424 mimics (Fig. 4B). E2F7, a transcription factor, was predicted to have a putative miR-424 binding site within its 3'UTR (Fig. 4C) and thus was selected for subsequent study. To confirm that miR-424 directly targets E2F7, we performed luciferase reporter assays to examine whether miR-424 interacts directly with its target E2F7. We constructed a series of 3'UTR fragments, including the full-length wild-type E2F7 3'UTR and a binding site mutant. These fragments were 
A

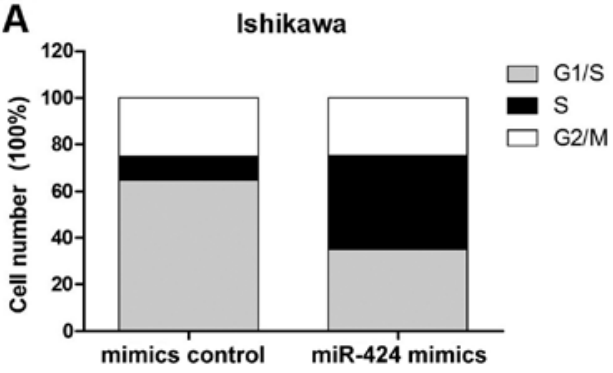

C

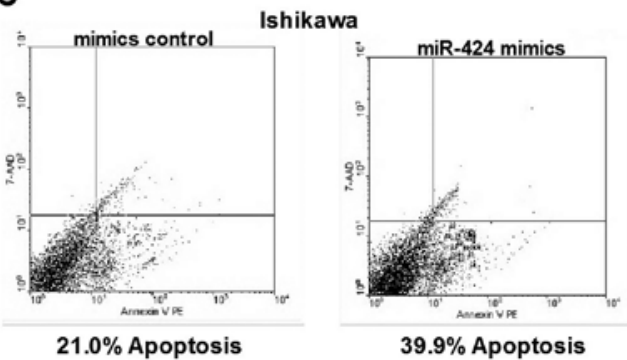

B

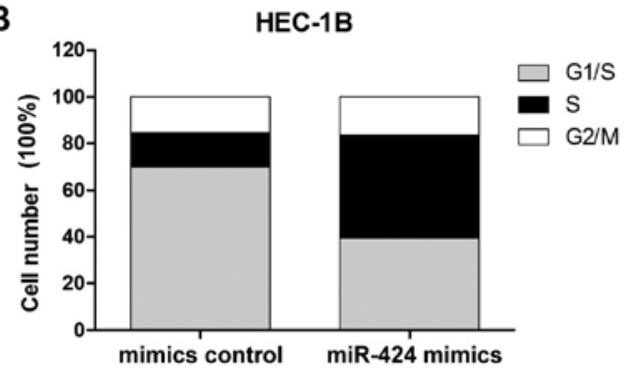

D

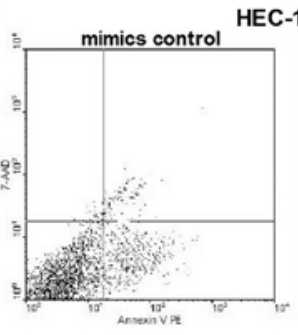

22.3\% Apoptosis

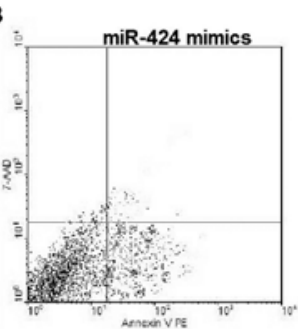

$45.2 \%$ Apoptosis

Figure 3. miR-424 initiates endometrial cancer cell line S-phase arrest and promotes apoptosis. (A and B) Flow cytometric analysis reveals S-phase arrest of Ishikawa and HEC-1B cells $48 \mathrm{~h}$ after transfection with miR-424-mimics compared to mimics control. (C and D) Incidence of apoptotic cells was studied by flow cytometry. The cells were stained with Annexin V-fluorescein isothiocyanate and counterstained with 7-ADD.

A

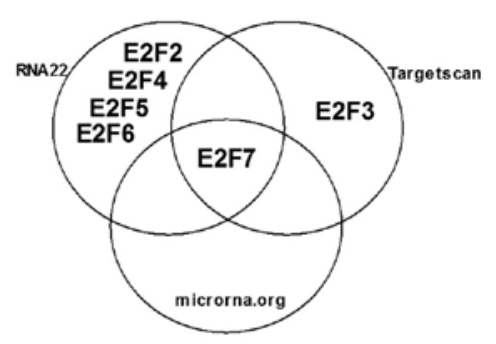

miR-424 target prediction
B

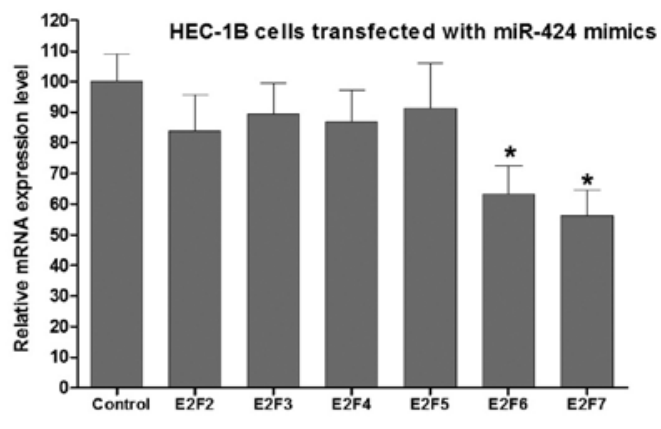

C

2580-----2586

E2F7-3'UTR WT 5'.AGGUAUUAAUUUUAUUGCUGCUU...

miR-424 3' AAGUUUUGUACUUAACGACGAC

\section{E2F7-3'UTR mut 5'.AGGUAUUAAUUUUAUUCGGCCUU...}

D

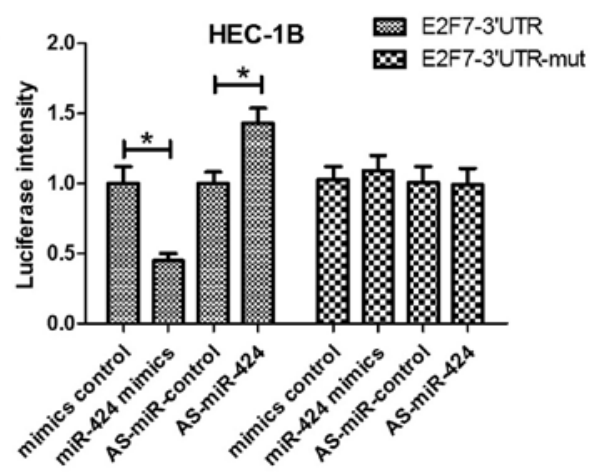

E
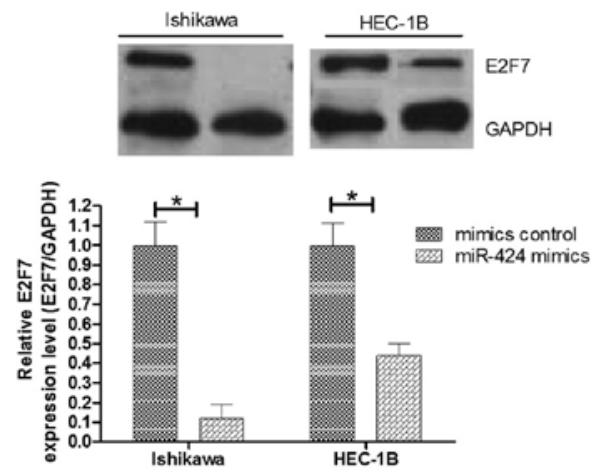

Figure 4. miR-424 directly targets transcription factor E2F7. (A) Potential target genes of miR-424 by bioinformatic analyses (TargetScan, RNA22 and microrna.org). (B) RT-PCR analysis indicated that E2F7 expression was significantly decreased in HEC-1B cells transfected with miR-424 mimics. ("P<0.05). (C) The predicted miR-424 binding site on the E2F7 mRNA 3'UTR. (D) HEC-1B cells were transfected with the wild-type (E2F7-3'UTR) or mutated version (E2F7-3'UTR-mut) of the luciferase-E2F7 3'-UTR reporter vector as well as the miR-424 mimics, mimics control or as-miR-424, as-miR-control. The miR424 mimics reduced the luciferase intensity from the luciferase-E2F7 3'-UTR reporter vector, while the as-miR-424 increased the luciferase intensity. The mutant E2F7 3'UTR did not alter the luciferase intensity. ("P<0.05). (E) Measurement of E2F7 expression levels by western blot analysis. Protein was extracted from Ishikawa and HEC-1B cells transfected with the miR-424 mimics or mimics control. The endogenous expression levels of the GAPDH protein were used for normalization, and the relative E2F7 protein expression levels are shown ("P<0.05). 

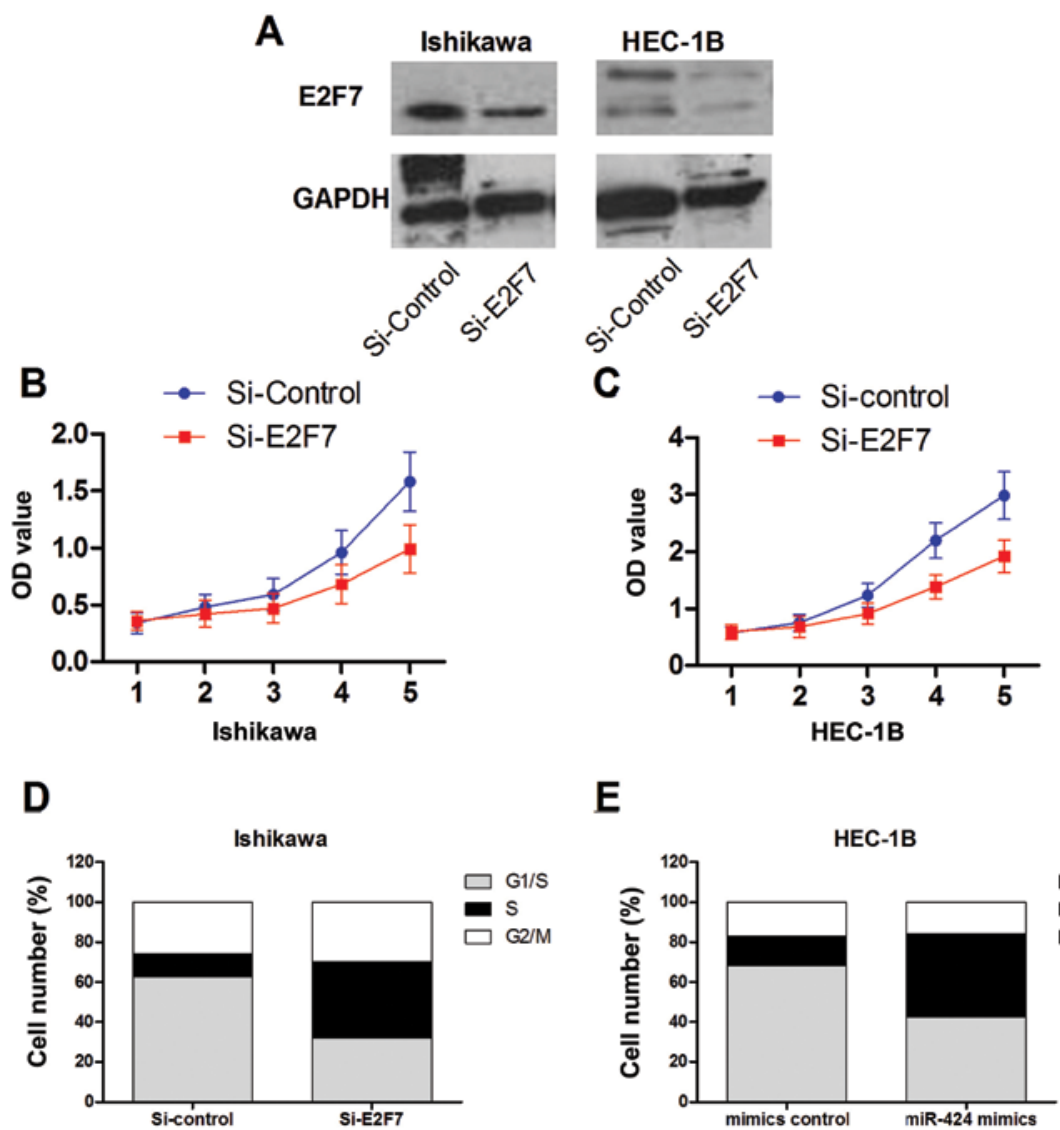

E
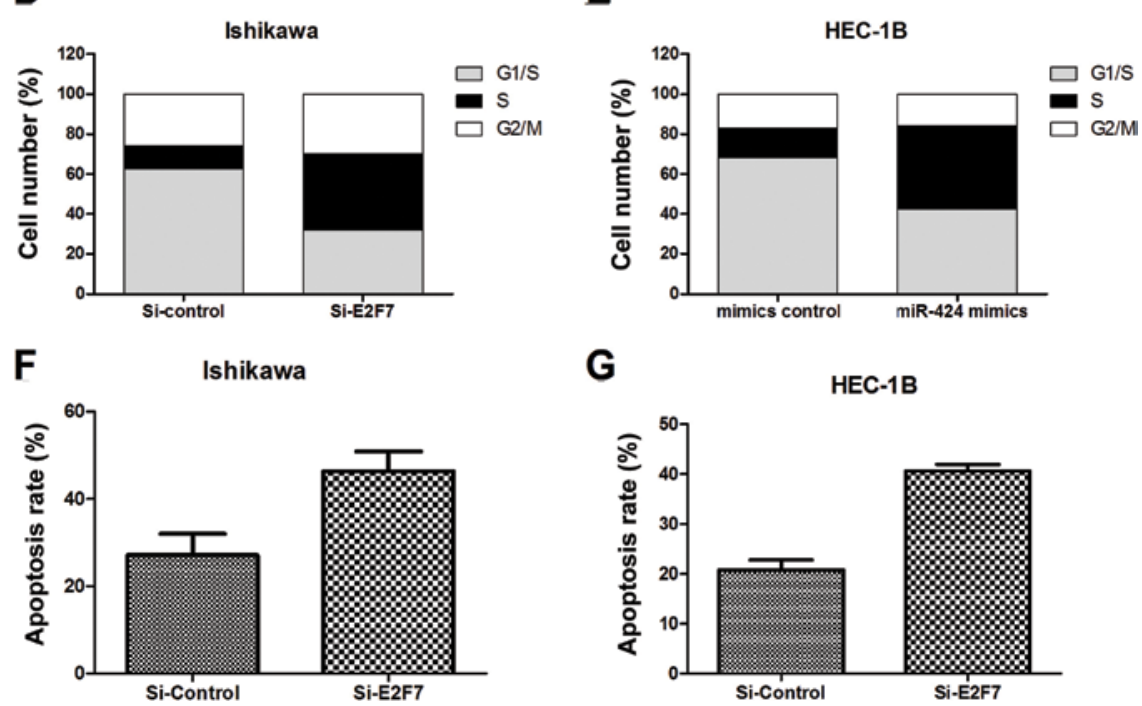

G

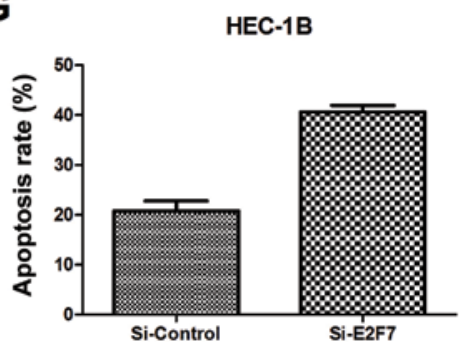

Figure 5. Knockdown of E2F7 inhibits the growth of endometrial cancer cells in vitro. (A) E2F7 protein expression levels were significantly decreased in Ishikawa and HEC-1B cells transfected with pSilencer/si-E2F7 (si-E2F7). (B and C) Measurement of cell growth by the MTT assay. Following transfection of Ishikawa and HEC-1B cells with pSilencer/si-E2F7 (si-E2F7) or control vector (si-control). The data present means \pm SD. (D and E) Flow cytometric analysis reveals S-phase arrest of Ishikawa and HEC-1B cells $48 \mathrm{~h}$ after transfection with pSilencer/si-E2F7 (si-E2F7) compared to control vector (si-control). (F and G) Incidence of apoptotic cells was studied by the fluorescence activated cell sorting.

then inserted into the pmirGLO luciferase reporter plasmid. In HEC-1B cells, we found that the co-transfection of miR-424 and the wild-type E2F7 3'UTR caused a significant decrease in luciferase units compared with the controls. The luciferase intensity was increased after transfected with the wild-type E2F7 3'UTR reporter vector as well as ASO-424 (as-miR-424). However, the co-transfection of the mutant E2F7 3'UTR and miR-424 mimics or ASO-424 (as-miR-424) did not alter the luciferase intensity (Fig. 4D). Furthermore, overexpression of miR-424 reduced the E2F7 protein expression in Ishikawa and HEC-1B cells (Fig. 4E). Taken together, these results suggest that miR-424 binds directly to the 3'UTR of E2F7, thereby repressing gene expression.

Knockdown of E2F7 inhibits the growth of endometrial cancer cells in vitro. E2F7 is a common transcription factor playing an essential role in the regulation of cell cycle progression. An RNAi approach was used to determine whether the knockdown of E2F7 affects cell growth. The siRNA expression vector, pSilencer/si-E2F7, effectively reduced E2F7 protein levels (Fig. 5A). The transient transfection of pSilencer/si-E2F7 into Ishikawa and HEC-1B cells indicated that knockdown of E2F7 could suppress cell growth in 5 days, as determined by the MTT assay (Fig. 5B and C). Flow cytometry revealed that the percentage of $S$ phase Ishikawa and HEC-1B cells markedly increased in the pSilencer/si-E2F7 transfected group, which was higher than that in $\mathrm{NC}$, suggesting that knockdown of E2F7 can initiate S-phase arrest (Fig. 5D and E). FACS analysis revealed that the depressed expression of E2F7 led to endometrial cancer cell apoptosis (Fig. 5F and G). These data demonstrated that knockdown of E2F7 initiates endometrial cancer cell line S-phase arrest and promotes apoptosis. 
A

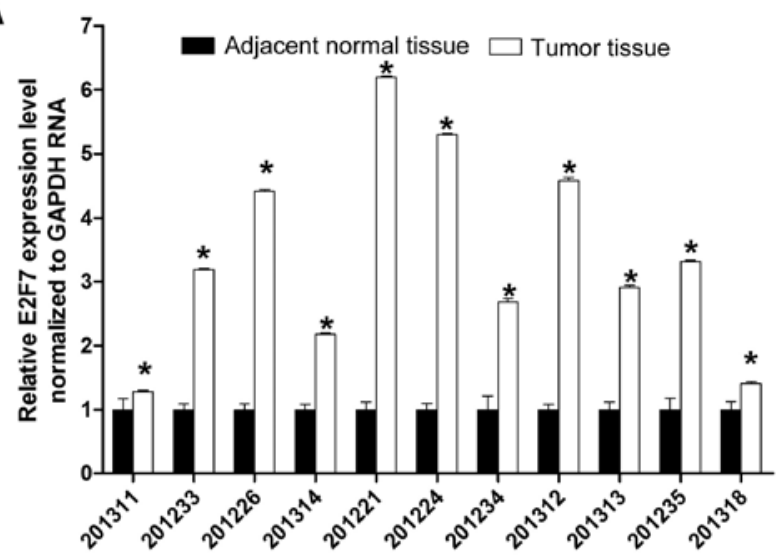

B

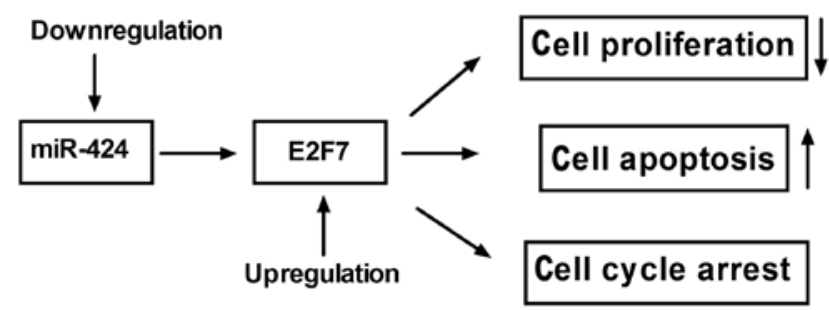

Figure 6. Quantitative analysis of E2F7 expression in endometrial cancer tissue. (A) Quantitative analysis of E2F7 expression in endometrial cancer tissues. To determine the expression of E2F7 in endometrial cancer tissues and adjacent normal tissues, a RT-PCR assay was performed on the 11 pairs of endometrial tissue samples, and the upregulation fold of E2F7 was shown $\left({ }^{*} \mathrm{P}<0.05\right)$. (B) Schematic depiction of miR-424-mediated repression of E2F7 in endometrial cancer cells that simultaneously suppresses cell proliferation, increases cell apoptosis and causes cell cycle arrest.

The results are consistent with the finding that miR-424 overexpression is able to suppress cell growth in vitro, which provides further evidence that E2F7 is involved in the miR424-mediated suppression of endometrial cancer. Accordingly, the identification of E2F7 as a target gene of miR-424 may explain, at least in part, the reason for miR-424 overexpression suppressing cell growth.

Quantitative analysis of E2F7 expression in endometrial cancer tissue. To determine the expression of E2F7 in endometrial cancer and adjacent normal tissue, RT-PCR for E2F7 was performed on 11 tissue pairs, each consisting of an endometrial cancer and adjacent normal tissue. In general, E2F7 expression levels were significantly higher in endometrial cancer tissues than in the matched normal tissues (Fig. 6A). By contrast, miR-424 expression levels were predominantly downregulated in endometrial cancer tissue (Fig. 1A and B).

\section{Discussion}

Identification of molecular and cellular mechanisms responsible for tumorigenesis and the progression of endometrial cancer are critical to the development of novel diagnostic and therapeutic strategies for endometrial cancer patients. miRNAs are considered new candidate therapeutic agents for endometrial cancer owing to their involvement in cancer initiation and progression (22). Currently, the regulation of
miRNAs is a topic that has garnered increasing attention, and some upregulated or downregulated miRNAs of endometrial cancer have been reported (14-20). However, the understanding of the aberrant expression and potential roles of miRNAs remains to be clarified. In 2012, Ramón et al reported that in 41 tissue samples of endometrial cancer patients, miR-15b, $-17-5 \mathrm{p},-20 \mathrm{a},-125 \mathrm{a},-214^{*},-221,-222$ and -424 were found to be significantly downregulated, compared with the miRNAs in the women without endometrial cancer. However, the exact mechanism of abnormal miRNAs in endometrial cancer were not showed (23). The present study identified that compared with normal samples, miR-424 was significantly downregulated in endometrial cancer samples, which is consistent with the study results of Ramón et al (23). To investigate the role of miR-424 in endometrial cancer, miR-424 mimics were used to enhance miR-424 expression in Ishikawa and HEC-1B cells. The results showed that overexpressed miR-424 suppressed the growth of Ishikawa and HEC-1B cells and induced endometrial cancer cell apoptosis. We used the luciferase reporter assay to confirm the target gene of miR-424 was E2F7, and found that pSilencer/si-E2F7 and the miR-424 mimics worked in an antagonistic way. In the present study, we confirmed that the miR-424-E2F7 signaling pathway is a functional mechanism by which miR-424 suppresses endometrial cancer. However, miRNAs usually function in the regulation of multiple targets, thus whether any other signalling pathway played a role via miR-424 in endometrial cancer was not determined.

For miR-424, our findings are consistent with studies on other types of malignancy, such as breast (24), liver (25), pancreas (26), prostate and colon (27), and bone cancer (28). Ruiz-Llorente et al reported that overexpression of miR-424 mimicked the repressive effect of T3 on cell proliferation, growth in suspension, migration and invasion (24). Yu et al found that miR-424 was involved in the tumorigenesis of hepatocellular carcinoma (HCC) by suppression of c-Myb. The expression of miR-424 was downregulated in HCC and the overexpression of miR-424 reduced HCC cell proliferation, migration and invasion (25). Similarly, downregulation of miR-424 was also detected in human prostate, colon and bone cancer tissue $(27,28)$. Exogenous miR-424/503 can significantly upregulate Rictor, an mTORC2-specific component, promote the formation of mTORC2 and induce activation of $\mathrm{mTORC} 2$, resulting in the promotion of tumor growth and invasion (27). In U2OS cells, miR-424 plays a key role in inhibiting osteosarcoma cell migration and invasion by targeting FASN (28).

The mammalian E2F family of transcription factors is crucial in the regulation of cell proliferation, apoptosis and differentiation. Our results demonstrated that E2F7 is a target of miR-424 in endometrial cancer. E2F7 has been found as a transcriptional repressor capable of negatively influencing cell proliferation (29). E2F7 is thought to be oncogenic and its expression is upregulated in acute myeloid leukemia (AML) and cutaneous squamous cell carcinomas (CSCC) $(30,31)$. E2F7 regulates cell cycle by repressing a subset of E2F target genes whose products are required for cell cycle progression (32). miR-424 inhibits cancer growth by blocking the expression and activity of transcription factor E2F7. The loss of miR-424 leads to the upregulation of E2F7, consequently resulting in malignant transformation. 
In summary, the present study indicates that miR-424 acts as a tumor suppressor by targeting E2F7 in endometrial cancer. Although there are some limitations to the present study, such as the relatively fewer numbers of endometrial cancer samples, and the lack of in vivo studies, our findings may aid the continued investigation of the regulation of miR-424 in endometrial cancer and its role as a potential biomarker as well as a novel therapeutic target of endometrial cancer, to demonstrate that miRNA-based clinical therapies are feasible options in the future.

\section{Acknowledgements}

The present study was supported by the Doctor Startup Foundation of Zunyi Medical College Guizhou Province in China [grant no. 2013(06)].

\section{References}

1. Pillai RS, Bhattacharyya SN and Filipowicz W: Repression of protein synthesis by miRNAs: how many mechanisms? Trends Cell Biol 17: 118-126, 2007.

2. Peters L and Meister G: Argonaute proteins: mediators of RNA silencing. Mol Cell 26: 611-623, 2007.

3. Kopnin BP: Targets of oncogenes and tumor suppressors: key for understanding basic mechanisms of carcinogenesis. Biochemistry 65: 2-27, 2000.

4. Steele CW, Oien KA, McKay CJ and Jamieson NB: Clinical potential of microRNAs in pancreatic ductal adenocarcinoma. Pancreas 40: 1165-1171, 2011.

5. Lujambio A, Calin GA, Villanueva A, Ropero S, et al: A microRNA DNA methylation signature for human cancer metastasis. Proc Natl Acad Sci USA 105: 13556-13561, 2008.

6. Felli N, Fontana L, Pelosi E, Botta R, et al: MicroRNAs 221 and 222 inhibit normal erythropoiesis and erythroleukemic cell growth via kit receptor down-modulation. Proc Natl Acad Sci USA 102: 18081-18086, 2005.

7. Li J, Huang H, Sun L, Yang M, et al: MiR-21 indicates poor prognosis in tongue squamous cell carcinomas as an apoptosis inhibitor. Clin Cancer Res 15: 3998-4008, 2009.

8. Dews M, Homayouni A, Yu D, Murphy D, et al: Augmentation of tumor angiogenesis by a Myc-activated microRNA cluster. Nat Genet 38: 1060-1065, 2006.

9. Chen CZ, Li L, Lodish HF and Bartel DP: MicroRNAs modulate hematopoietic lineage differentiation. Science 303: 83-86, 2004.

10. Ma L, Young J, Prabhala H, Pan E, et al: miR-9, a MYC/MYCNactivated microRNA, regulates E-cadherin and cancer metastasis. Nat Cell Biol 12: 247-256, 2010.

11. DeSantis CE, Lin CC, Mariotto AB, Siegel RL, et al: Cancer treatment and survivorship statistics, 2014. CA Cancer J Clin 64: 252-271, 2014.

12. Banno K, Nogami Y, Kisu I, Yanokura M, et al: Candidate biomarkers for genetic and clinicopathological diagnosis of endometrial cancer. Int J Mol Sci 14: 12123-12137, 2013.

13. Mozos A, Catasús L, D'Angelo E, et al: The FOXO1-miR27 tandem regulates myometrial invasion in endometrioid endometrial adenocarcinoma. Hum Pathol 45: 942-951, 2014.

14. Huang YW, Kuo CT, Chen JH, et al: Hypermethylation of miR-203 in endometrial carcinomas. Gynecol Oncol 133: 340-345, 2014.
15. Zhang G, Hou X, Li Y and Zhao M: MiR-205 inhibits cell apoptosis by targeting phosphatase and tensin homolog deleted on chromosome ten in endometrial cancer Ishikawa cells. BMC Cancer 14: 440, 2014.

16. Wang Y, Adila S, Zhang X, Dong Y, et al: MicroRNA expression signature profile and its clinical significance in endometrioid carcinoma. Zhonghua Bing Li Xue Za Zhi 43: 88-94, 2014 (In Chinese).

17. Kong X, Xu X, Yan Y, et al: Estrogen regulates the tumour suppressor miRNA-30c and its target gene, MTA-1, in endometrial cancer. PLoS One 9: e90810, 2014.

18. Konno Y, Dong P, Xiong Y,Suzuki F, et al: MicroRNA-101 targets EZH2, MCL-1 and FOS to suppress proliferation, invasion and stem cell-like phenotype of aggressive endometrial cancer cells. Oncotarget 5: 6049-6062, 2014.

19. Ye W, Xue J, Zhang Q, et al: MiR-449a functions as a tumor suppressor in endometrial cancer by targeting CDC25A. Oncol Rep 32: 1193-1199, 2014.

20. Lee H, Park CS, Deftereos G, Morihara J, et al: MicroRNA expression in ovarian carcinoma and its correlation with clinicopathological features. World J Surg Oncol 10: 174, 2012.

21. Ratner ES, Tuck D, Richter C, Nallur S, et al: MicroRNA signatures differentiate uterine cancer tumor subtypes. Gynecol Oncol 118: 251-257, 2010

22. Umene K, Banno K, Kisu I, Yanokura M, et al: New candidate therapeutic agents for endometrial cancer: Potential for clinical practice (Review). Oncol Rep 29: 855-860, 2013.

23. Ramón LA, Braza-Boïls A, Gilabert J, Chirivella M, et al: microRNAs related to angiogenesis are dysregulated in endometrioid endometrial cancer. Hum Reprod 27: 3036-3045, 2012.

24. Ruiz-Llorente L, Ardila-González S, Fanjul LF, MartínezIglesias $\mathrm{O}$ and Aranda A: microRNAs 424 and 503 are mediators of the anti-proliferative and anti-invasive action of the thyroid hormone receptor beta. Oncotarget 5: 2918-2933, 2014

25. Yu L, Ding GF, He C, Sun L, Jiang Y and Zhu L: MicroRNA-424 is down-regulated in hepatocellular carcinoma and suppresses cell migration and invasion through c-Myb. PLoS One 9: e91661, 2014.

26. Wu K, Hu G, He X, Zhou P, et al: MicroRNA-424-5p suppresses the expression of SOCS6 in pancreatic cancer. Pathol Oncol Res 19: 739-748, 2013.

27. Oneyama C, Kito Y, Asai R, Ikeda J, et al: miR-424/503-mediated Rictor upregulation promotes tumor progression. PLoS One 8: e80300, 2013.

28. Long XH, Mao JH, Peng AF, Zhou Y, Huang SH and Liu ZL: Tumor suppressive microRNA-424 inhibits osteosarcoma cell migration and invasion via targeting fatty acid synthase. Exp Ther Med 5: 1048-1052, 2013.

29. de Bruin A, Maiti B, Jakoi L, Timmers C, Buerki R and Leone G: Identification and characterization of $E 2 F 7$, a novel mammalian E2F family member capable of blocking cellular proliferation. J Biol Chem 278: 42041-42049, 2003.

30. Salvatori B, Iosue I, Mangiavacchi A, Loddo G, et al: The microRNA-26a target E2F7 sustains cell proliferation and inhibits monocytic differentiation of acute myeloid leukemia cells. Cell Death Dis 3: e413, 2012

31. Endo-Munoz L, Dahler A, Teakle N, Rickwood D, et al: E2F7 can regulate proliferation, differentiation, and apoptotic responses in human keratinocytes: implications for cutaneous squamous cell carcinoma formation. Cancer Res 69: 1800-1808, 2009.

32. Di Stefano L, Jensen MR and Helin K: E2F7, a novel E2F featuring DP-independent repression of a subset of E2F-regulated genes. EMBO J 22: 6289-6298, 2003. 